\title{
Severidad de la preeclampsia y su relación con volumen plaquetario y ancho de distribución eritrocitaria
}

Rosas-Cabral Alejandro*, Viana-Rojas Jesús Antonio*, Terrones-Saldívar Ma del Carmen*, Prieto-Macías Jorge**, Gutiérrez-Campos Rafael*, Pérez-Ramírez Oscar de Jesús***, Hernández-González Flavio*, Uria-Torrijas Tania*, Serrano-Díaz Leopoldo César***

\section{Resumen}

- Objetivo: Se ha establecido la asociación entre el ancho de dis-

- tribución eritrocitario (ADE) y el volumen plaquetario medio

- (VPM) con la hipertensión arterial sistémica. Sin embargo, se

- han realizado pocos estudios en pacientes con preeclampsia,

- obteniéndose resultados poco consistentes. Nuestro objetivo fue

- evaluar la relación entre el ADE y el VPM con la severidad de la

- preeclampsia. Métodos: Incluimos 81 pacientes con preeclamp-

- sia (3I leve, 50 severa) y 80 pacientes con embarazo normo-

- tenso. Los datos clínicos, características socio-demográficas y

- valores de laboratorio, incluyendo ADE y VPM de cada paciente

- fueron registrados. Resultados: Las medidas de hemoglobina y

- conteo plaquetario fueron similares entre ambos grupos. Las

- pacientes con preeclampsia tuvieron niveles de ADE (1 $4.6 \pm$

- 1.3 vs I $3.4 \pm 0.7, p=0.000$ I) y VPM (II.7 \pm 3.0 vs $10.9 \pm$

- I.3, $p=0.03$ ) más elevados que el grupo control. El subgrupo de

- preeclampsia severa tuvo niveles más elevados de ADE (I 5.0

- \pm I.I vs $13.88 \pm 0.79, p=0.001$ ) y VPM (I $2.7 \pm 2.8$ vs 10.45

$\pm 2.4, p=0.01)$ que las pacientes con preeclampsia leve. Con-

- clusión: En este trabajo demostramos que el ADE y el VPM son

- medidas accesibles asociadas con la severidad de la preeclamp-

sia. LUXMÉDICA, AÑO 13, NÚMERO 38, MAY-AGO 2018 PP 9-17.

Palabras clave: ancho de distribución eritrocitaria, volumen plaquetario medio, preeclampsia.

\section{Abstract}

Objective: The association between erythrocyte distribution width (EDW) and mean platelet volume (MPV) with systemic hypertension has been established. However, few studies have been performed in patients with preeclampsia, obtaining inconsistent results. Our objective was to evaluate the relationship between EDW and MPV with the severity of preeclampsia. Methods: We included 81 patients with preeclampsia (31 mild, 50 severe) and 80 patients with normotensive pregnancy. Clinical data, socio-demographic characteristics and laboratory values, including EDW and MPV of each patient, were recorded.Results: The measurements of hemoglobin and platelet count were similar between both groups. Patients with preeclampsia had EDW levels $(14.6 \pm 1.3 \mathrm{vs} / 3.4 \pm 0.7, p=0.0001)$ and MPV (I I.7 \pm 3.0 vs $10.9 \pm 1.3, p=0.03$ ) higher than the control group. The subgroup of severe preeclampsia had higher EDW levels (15.0 \pm 1.1 vs $13.88 \pm 0.79, p=0.001)$ and MPV $(12.7 \pm 2.8$ vs $10.45 \pm 2.4, p=0.01)$ than patients with mild preeclampsia. Conclusion: In this work, we show that EDW and MPV are accessible measures associated with the severity of preeclampsia. LUXMÉDICA, AÑO 13, NÚMERO 38, MAY-AG0 2018 PP 9-17.

Keywords: erythrocyte distribution width, mean platelet volume, preeclampsia

Investigadores del Departamento de Medicina del Centro de Ciencias de la Salud de la Universidad Autónoma de Aguascalientes, Aguascalientes, México.

** Médico Adscrito a la Unidad de Terapia Intensiva, Hospital General de Zona Nº1. IMSS, Aguascalientes, Aguascalientes, México.

*** Jefe de la Clínica de Embarazo de Alto Riesgo, Hospital de la Mujer, Aguascalientes, Aguascalientes, México.

***** Coordinador del Departamento de Hematología. Facultad de Medicina de la Universidad Autónoma de San Luis Potosí.

Fecha de recibido: 8 de febrero 2018

Fecha de aceptación: 5 de marzo 2018

Correspondencia: Dr Alejandro Rosas Cabral. Departamento de Medicina, edificio 107, planta alta. Centro de Ciencias de la Salud. Universidad Autónoma de Aguascalientes Av. Universidad \#940 Ciudad Universitaria, Código postal 20131. Aguascalientes, Ags. México. Teléfono y fax: 01449 9108440 Correo electrónico: drrosascabral@gmail.com 


\section{Introducción}

La preeclampsia es un desorden multifactorial del embarazo caracterizado por hipertensión arterial y proteinuria, ${ }^{1}$ afecta al 5 a $7 \%$ de las mujeres embarazadas cada año ${ }^{2}$ y es la principal causa de mortalidad materna y perinatal en el mundo. ${ }^{3}$ Aunque la causa precisa de la preeclampsia es aún desconocida, se han identificado algunos factores relevantes para su patogenia, como son: el estrés oxidativo, la inflamación, la disfunción endotelial, placentación deficiente, así como factores inmunológicos y antiangiogénicos. ${ }^{2,4,5}$

El ancho de distribución eritrocitario (ADE) —coeficiente de variación del tamaño de los eritrocitos-y el volumen plaquetario medio (VPM) - promedio del tamaño de las plaquetas- son reportados de manera rutinaria como parte de la biometría hemática. Aunque son empleados comúnmente en el diagnóstico diferencial de la anemia y trombocitopenia, recientemente han sido estudiados como marcadores asociados con la mortalidad cardiovascular en pacientes con insuficiencia cardíaca, angina inestable, infarto cardíaco, evento cerebrovascular y en la población general. ${ }^{6-10}$

Se ha establecido una fuerte asociación entre el ADE y el VPM con la hipertensión arterial sistémica en pacientes sin embarazo; $;^{11-14}$ sin embargo, pocos estudios han sido realizados en pacientes con preeclampsia, obteniéndose resultados contradictorios. ${ }^{15-18}$ El propósito de este estudio es aumentar el conocimiento acerca de esta asociación, por tal razón, nosotros investigamos la relación entre el ADE y VPM con la severidad de la enfermedad en pacientes con preeclampsia.

\section{| | | | | | | | | | | | | | | | | | | | | | | | | | | | | | | | | | | | | | | | | | | | | | | | | | | | | | | | | | | | | | | | | | | | | | | | | | | | | | | | | | | | | | | | | | | | | | | | | | | | | | | ||}

\section{Material y métodos}

\section{Población en estudio}

Se realizó un estudio transversal y analítico de casos y controles. De junio de 2015 a junio de 2017, incluimos a 80 pacientes con preeclampsia y 80 pacientes con embarazo normotenso. El diagnóstico de preeclampsia fue realizado con base en los criterios del American College of Obstetricians and Gynecologists del 2002: ${ }^{19}$ dos mediciones de tensión arterial sistólica $\geq 140 \mathrm{mmHg}$ o de tensión arterial diastólica $\geq 90 \mathrm{mmhg}$ tomadas con 4 horas de diferencia y una proteína en orina de $24 \mathrm{hrs} \geq 300 \mathrm{mg}$. Clasificamos un caso como preeclampsia severa si cumplía alguno de los siguientes criterios: tensión arterial $\geq 160 / 110 \mathrm{mmHg}$, edema pulmonar, incremento al doble de la creatinina sérica, oliguria, cuenta plaquetaria $<100,000$ eclampsia, restricción en el crecimiento intrauterino u oligohidramnios. Las pacientes con preeclampsia que no cumplieron ninguno de estos criterios fueron clasificadas como preeclampsia leve. Las pacientes con embarazo múltiple, diabetes mellitus, enfermedad renal, enfermedades autoinmunes, infecciones agudas o crónicas, neoplasias con metástasis a médula ósea, anemia, hemólisis, enfermedades hematológicas, tiroideas y hepáticas conocidas fueron excluidas. El grupo control consis- 
tió en pacientes con embarazo normotenso que cursaban la segunda mitad de su embarazo sin complicaciones. El comité de ética local proporcionó la aprobación del estudio.

\section{Recolección de los datos y toma de muestras de laboratorio}

Se evaluó el expediente médico de cada paciente a su ingreso al hospital (casos) o su consulta prenatal (controles) para obtener información relevante sobre su historial obstétrico. Se registró la edad gestacional de cada paciente al momento de la toma de sus exámenes de laboratorio. La medición de la tensión arterial fue realizada al ingreso al hospital (casos) o durante la consulta prenatal (controles). En pacientes con preeclampsia, esta medición fue realizada en dos ocasiones separadas por un período de cuatro horas; se registró la cifra más elevada.

Se obtuvo sangre venosa de todas las pacientes en el estudio. La medición de hemoglobina, volumen globular medio, ancho de distribución eritrocitario, conteo plaquetario, volumen plaquetario medio, ácido úrico, creatinina, albúmina y proteí- na en orina de 24 horas fueron llevadas a cabo por el laboratorio de nuestra institución utilizando métodos estandarizados.

\section{Análisis estadístico}

Las variables continuas son expresadas como promedio y desviación estándar $( \pm D E)$ en caso de seguir una distribución paramétrica; y como mediana y rango, en caso de seguir una distribución no paramétrica. Las variables categóricas son expresadas en frecuencia y porcentaje (\%). Realizamos la comparación de valores continuos entre dos grupos con la prueba $t$ de Student o con la prueba de $U$ de Mann-Whitney según correspondiera. Las variables categóricas fueron comparadas usando la prueba de Chi-cuadrada.

Finalmente, realizamos un análisis de curva ROC (Receiver Operating Characteristic) con el fin de identificar un punto de corte efectivo para el valor de ADE y VPM como predictores de preeclampsia severa.

El análisis estadístico se llevó a cabo con el paquete GraphPad Prism versión 5.0 para Windows. ${ }^{20}$ Un valor de $p<0.05$ fue considerado como estadísticamente significativo.

\section{| | | | | | | | | | | | | | | | | | | | | | | | | | | | | | | | | | | | | | | | | | | | | | | | | | | | | | | | | | | | | | | | | | | | | | | | | | | | | | | | | | | | | | | | | | | | | | | | | | | | | | | |}

\section{Resultados}

\section{Comparación entre pacientes con preeclampsia y embarazo normotenso}

Se incluyeron en total 81 pacientes con preeclampsia y 80 pacientes con embarazo normotenso. En la tabla 1, se pueden observar las características iniciales de las pacientes en estudio. No se encontraron diferencias significativas entre los dos grupos en relación a las gestas, partos, cesáreas, abortos, hemoglobina y cuenta de plaquetas. No obstante, al comparar el grupo control con el grupo de pacientes con preeclampsia, éstas últimas, tuvieron cifras de tensión arterial sistólica, tensión arterial diastólica, ADE, VPM y edad significativamente más elevadas. Otras características estudiadas no tuvieron diferencias estadísticamente significativas entre ambos grupos. 


\section{Tabla I}

Características basales de 161 pacientes con preeclampsia y embarazo normal.

\begin{tabular}{|lccc|}
\hline Parámetro & Casos & Controles & P \\
\hline & $(\mathrm{n}=81)$ & $(\mathrm{n}=80)$ & \\
\hline Edad en años (DE) & $26.0(6.6)$ & $23.0(5.6)$ & 0.002 \\
\hline Primigestas (\%) & $46(56.7)$ & $41(51.2)$ & 0.87 \\
\hline Gestaciones (rango) & $2(1-6)$ & $1(1-3)$ & 0.97 \\
\hline Partos (rango) & $0(0-3)$ & $0(0-1)$ & 0.75 \\
\hline Cesáreas (rango) & $1(0-3)$ & $0(0-2)$ & 0.99 \\
\hline Abortos (rango) & $0(0-2)$ & $0(0-1)$ & 0.64 \\
\hline Edad gestacional & & $13(16.3)$ & \\
\hline 20-34 SDG (\%) & $67(83.7)$ & 0.12 \\
\hline $34.1-42$ SDG (\%) & $26(32.0)$ & $107.5(9.0)$ & 0.0001 \\
\hline TA Sistólica en mm Hg (DE) & $55(68.0)$ & $67.5(9.6)$ & 0.0001 \\
\hline TA Diastólica en mm Hg (DE) & $171.2(23.9)$ & $12.9(0.8)$ & 0.86 \\
\hline Hb en g/dL (DE) & $111.7(15.8)$ & $88.9(4.1)$ & 0.12 \\
\hline VGM en fl (DE) & $12.9(1.1)$ & $221,000(69)$ & 0.15 \\
\hline Plaquetas en cel /mm3 (DE) & $87.9(2.8)$ & $13.4(0.7)$ & 0.0001 \\
\hline ADE en \% (DE) & $218,000(70)$ & $10.9(1.3)$ & 0.03 \\
\hline VPM en fl (DE) & $14.6(1.3)$ & & \\
\hline
\end{tabular}

Las variables categóricas son reportadas como número (\%). Las variables continuas son reportadas como promedio (Desviación Estándar) y mediana (rango) según su normalidad, $S D G=$ semanas de gestación; TA = tensión arterial; $\mathrm{mm} \mathrm{Hg}=$ milímetros de mercurio; $H b=$ hemoglobina $; V G M=$ volumen globular medio; $A D E=$ ancho de distribución eritrocitario; $V P M=$ volumen plaquetario medio.

\section{Comparación entre pacientes con preeclampsia leve y severa}

Al estudiar de manera separada a las pacientes con preeclampsia, el $59 \%$ presentó preeclampsia severa. Al analizar ambos subgrupos, no encontramos diferencias significativas en lo referido a la edad, gestas, partos, cesáreas, abortos, hemoglobi- na, cuenta de plaquetas, creatinina o albúmina; sin embargo, en las pacientes con preeclampsia severa encontramos valores significativamente mayores de ADE, VPM, ácido úrico, proteinuria y tensión arterial sistólica (tabla 2, figura 1). 


\section{Tabla 2}

Características basales de 81 pacientes con preeclampsia leve y severa.

\begin{tabular}{|lccc|}
\hline Parámetro & Leve $(\mathbf{n}=\mathbf{3 1})$ & Severa $(\mathbf{n}=\mathbf{5 0})$ & P \\
\hline Edad en años (DE) & $25.58(6.7)$ & $26.93(6.5)$ & 0.37 \\
\hline Primigestas (\%) & $14(56)$ & $17(45.6)$ & 1.00 \\
\hline Gestaciones (rango) & $1(1-3)$ & $2(1-6)$ & 0.95 \\
\hline Partos (rango) & $0(0-1)$ & $0(0-6)$ & 0.95 \\
\hline Cesáreas (rango) & $0(0-1)$ & $0(0-2)$ & 0.73 \\
\hline Abortos (rango) & $0(0-1)$ & $0(0-1)$ & 0.76 \\
\hline Edad gestacional & & $9(18)$ & 0.54 \\
\hline 20-34 SDG (\%) & $41(82)$ & 0.0001 \\
\hline 34.1-42 SDG (\%) & $11(36)$ & $181.22(24)$ & 0.004 \\
\hline TA Sistólica en mm Hg (DE) & $20(64)$ & $119.84(11.4)$ & 0.96 \\
\hline TA Diastólica en mm Hg (DE) & $153.2(8.5)$ & $12.9(1.2)$ & 0.39 \\
\hline Hb en g/dL (DE) & $103.55(6.5)$ & $88.7(3.6)$ & 0.44 \\
\hline VGM en fl (DE) & $12.9(1.1)$ & $213,900(76)$ & 0.001 \\
\hline Plaquetas cel/mm3 (DE) & $87.5(4.6)$ & $15.0(1.1)$ & 0.01 \\
\hline ADE en \% (DE) & $198,350(96)$ & $12.7(2.8)$ & 0.03 \\
\hline VPM en fl (DE) & $13.88(0.79)$ & $6.3(1.5)$ & 0.18 \\
\hline Ácido úrico en mg/dl (DE) & $10.45(2.4)$ & $0.9(0.8)$ & 0.01 \\
\hline Creatinina en mg/dl (DE) & $5.2(1.1)$ & $1779(1258)$ & 0.39 \\
\hline Proteinuria 24 h en mg/dl (DE) & $0.6(0.3)$ & $2.8(0.5)$ & \\
\hline Albúmina en mg/dl (DE) & $905(753)$ & & \\
\hline
\end{tabular}

Las variables categóricas son reportadas como número (\%). Las variables continuas son reportadas como promedio (DE) y mediana (rango) según su normalidad, $S D G=$ semanas de gestación; $T A=$ tensión arterial; $\mathrm{mmHg}=$ milimetros de mercurio; $H b=$ hemoglobina $; V G M=$ volumen globular medio; $A D E=$ ancho de distribución eritrocitario; VPM = volumen plaquetario medio; $h=$ horas

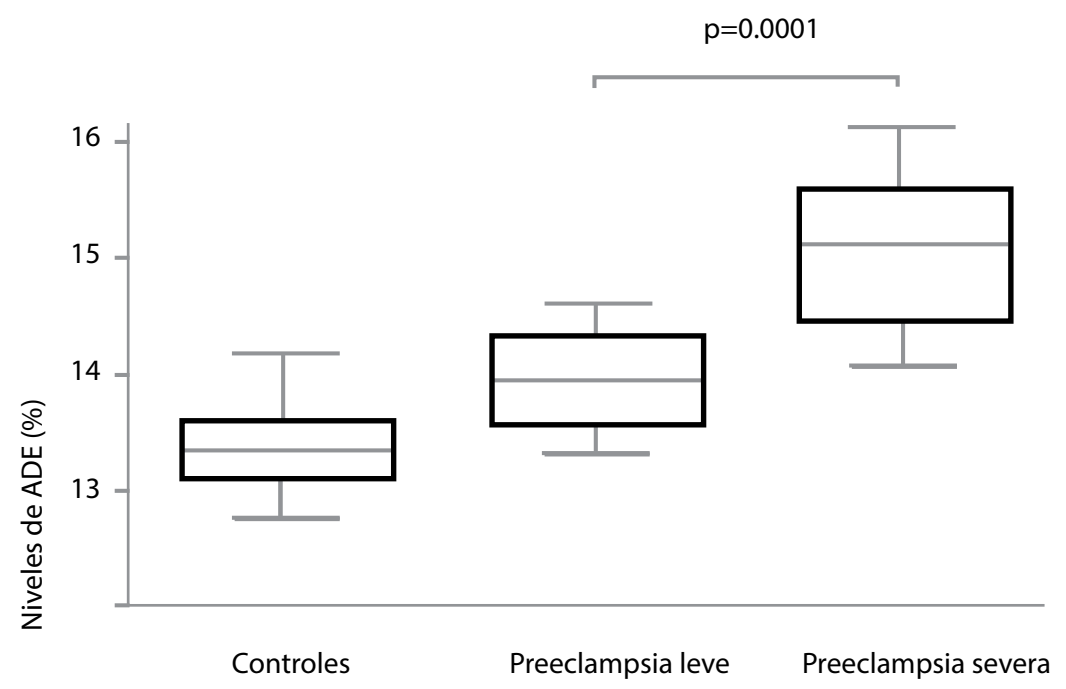

Figura 1 Comparación de los niveles de ancho de distribución eritrocitario (ADE) en pacientes con preeclamsia leve o severa y pacientes con embarazo normotenso. 


\section{Valor predictor del ancho de distribución eritrocitario y volumen plaquetario medio}

Construimos una curva ROC y en el análisis de la misma, encontramos que un valor de ADE de 14.95 es útil como un punto de corte para definir la severidad en pacientes con preeclampsia (área bajo la cur- va $=0.78 ;$ IC 95\%: 0.62-0.94). De igual forma, identificamos que un valor de VPM de 11.45 fue el punto de corte más efectivo (área bajo la curva $=0.76$; IC 95\%: 0.59-0.94). La razón de verosimilitud, sensibilidad y especificidad de los puntos de corte propuestos para el ADE y el VPM por nosotros se muestran en la tabla 3.

\section{Tabla 3}

\section{Análisis de curva ROC para el ADE y VPM}

\begin{tabular}{|l|l|l|l|l|l|l|}
\hline Parámetro & Punto de corte & $\begin{array}{l}\text { Sensibilidad } \\
\text { (IC 95\%) }\end{array}$ & $\begin{array}{l}\text { Especificidad } \\
\text { (IC 95\%) }\end{array}$ & $\begin{array}{l}\text { Razón de } \\
\text { verosimilitud }\end{array}$ & $\begin{array}{l}\text { Área bajo la } \\
\text { curva }\end{array}$ & P \\
\hline ADE (\%) & 14.95 & $50(25-75)$ & $93.7(70-99)$ & 8.0 & 0.78 & 0.005 \\
\hline VPM (fl) & 11.45 & $75(48-93)$ & $81.2(54-96)$ & 4.0 & 0.76 & 0.009 \\
\hline
\end{tabular}

La sensibilidad y especificidad son reportadas en porcentaje, $R O C=$ receiver operating characteristic; $A D E$ $=$ ancho de distribución eritrocitario; $V P M=$ volumen plaquetario medio; IC = Intervalo de confianza .

\section{Discusión}

Los niveles elevados de ADE son reflejo de una gran heterogeneidad en el tamaño de los eritrocitos, lo cual es causado por una perturbación en la degradación o maduración de los glóbulos rojos. El ADE es comúnmente usado en la práctica clínica para discriminar y diferenciar entre los diferentes tipos de anemia. ${ }^{21,22}$ Sin embargo, recientemente se ha relacionado con la presencia y severidad de la hipertensión. ${ }^{11,12}$ [0]Aunque, recientemente, también ha sido establecida en pacientes con infarto cardíaco, ${ }^{6}$ angina inestable, ${ }^{7}$ insuficiencia cardiaca aguda ${ }^{23}$ y enfermedad vascular cerebral. ${ }^{8} \mathrm{El}$ mecanismo de esta asociación aún es desconocido, pero la teoría inflamatoria es la más reconocida. La inflamación favorecería niveles elevados de ADE, debido a bloqueo reticuloendotelial, lo que disminuiría la disponibilidad del hierro para la eritropoyesis, suprimiendo la maduración de los eritrocitos y disminuyendo su vida media. $^{24}$
El VPM es comúnmente usado durante el diagnóstico diferencial de los diferentes tipos de trombocitopenia. ${ }^{25}$ Recientemente también ha sido relacionado con la presencia y severidad de la hipertensión. ${ }^{13}$ EI VPM es un marcador de la actividad plaquetaria y está asociado con la mortalidad cardiovascular. Al igual que el ADE, el mecanismo de esta asociación es inflamatorio. Estudios previos han demostrado que la elevación de citoquinas pro-inflamatorias conduce a la producción de plaquetas más grandes y reactivas; ${ }^{27}$ estas plaquetas se agregan más rápidamente, tienen mayores niveles de tromboxano $\mathrm{A} 2$ y expresan más receptores de glucoproteína IIb/IIla. ${ }^{27,28}$

Se ha considerado que la patogénesis de la preeclampsia incluye dos etapas claramente discernibles: una que inicia temprano en el embarazo involucrando una primera etapa subclínica y asintomática de placentación deficiente, producida por un remodelamiento deficiente de las arterias espirales; ${ }^{29}$ y en la segunda etapa, la 
respuesta sistémica en la madre se desencadenaría por la liberación de sustancias (inflamatorias, oxidativas o anti-angiogénicas) por la placenta hacia la circulación materna -como resultado de la hipoxia placentaria-29. Algunos investigadores han documentado que la perfusión uterina reducida (hipoxia placentaria) es capaz de generar la secreción de moléculas proinflamatorias dañando las células endoteliales maternas ${ }^{30}$. En la actualidad, se han encontrado niveles aumentados de marcadores de inflamación (citoquinas) en pacientes con preeclampsia en comparación con embarazos normotensos; ${ }^{31-33}$ y se ha observado que citoquinas como la IL-6 y el TNF-alfa se correlacionan de forma directa con la severidad de la enfermedad. ${ }^{34}$

La relación entre el ADE y el VPM con la preeclampsia ha sido investigada hasta hace poco, encontrándose resultados inconsistentes. Mientras que los estudios liderados por Kurt, Freitas y Yilmaz reportan una relación positiva con la preeclampsia severa, ${ }^{13,16,35}$ aquellos liderados por Abdullahi y Ceyhan descartan esta asociación. ${ }^{14,15}$ En nuestro estudio, encontramos niveles más elevados de ADE y VPM en pacientes con preeclampsia en comparación con embarazos normotensos. Esta diferencia fue mayor en el subgrupo de pacientes con preeclampsia severa. Además, identificamos puntos de corte predictores de preeclampsia severa para los parámetros estudiados, y que son similares a los reportados recientemente por Sen$\mathrm{Yu}$ y colaboradores, quienes refiere que un RDW de 14.1 tiene una sensibilidad $y$ especificidad del $72.5 \%$ y $77.9 \%,{ }^{36}$ para detectar preeclampsia, indudablemente se requieren de un mayor número de estudios para corroborar estos hallazgos. Lo anterior también va en línea con el he- cho de que recientemente se ha valorado la importancia de la variabilidad biológica de estos parámetros hematológicos (RDW, VPM), para incrementar la capacidad de discernimiento de los clínicos con el uso de estos valores. ${ }^{37,38}$

Algunas limitaciones de este estudio incluyen el número pequeño de pacientes y su diseño transversal, mediante el cual obtuvimos resultados basados en una determinación única de laboratorio, lo que podría no reflejar el estado clínico de los pacientes durante periodos más extensos. Además, no medimos marcadores de inflamación, hierro sérico, ácido fólico ni vitamina B12.

La identificación de marcadores pronósticos novedosos puede mejorar la toma de decisiones en las pacientes con preeclampsia. El ADE y el VPM podrían proveer información pronóstica relevante para mejorar la estratificación del riesgo de estas pacientes, aunado al hecho de que están ampliamente disponibles y son de muy bajo costo. Sin embargo, existe la necesidad de estudios prospectivos que permitan validar los puntos de corte propuestos y determinar el valor pronóstico de ambos marcadores.

En conclusión, el ADE y el VPM son marcadores relacionados con la severidad de la preeclampsia. Se encuentran ampliamente disponibles y su uso no representa un gasto adicional. Debido a estas ventajas, el ADE y VPM podrían ser usados en un futuro para estratificar el riesgo de los pacientes con preeclampsia.

\section{Conflictos de interés}

Los autores declaran no tener conflictos de interés. 


\section{Bibliografía}

1. ACOG Practice Bulletin. Diagnosis and management of preeclampsia and eclampsia. ACOG Committee on Practice Bulletins-Obstetrics. Obstet Gynecol 2002; 99(1):159-167.

2. Cornelius DC. Preeclamspia: From inflammation to immunoregulation. Clin Med Insights Blood Disord. 2018; Jan 10:10;11:179545417752325

3. Sibai BM. Preeclampsia as a causa of preterm and late preterm (near-term) births. Semin Perinatol 2006; 30:16-19.

4. Kim SM, Kim JS. A Review of mechanisms of implantation. Dev Reprod. 2017; 21(4):351-359.

5. Kenny LC, Kell DB. Immunological tolerance, pregnancy and preeclampsia: The roles of semen microbes and the father. Front Med. 2017;4:239

6. Dabbah S, Hammerman H, Markiewicz W, Aronson $D$. Relation between red cell distribution width and clinical outcomes after acute myocardial infarction. Am J Cardiol 2010; 105(3):312-7.

7. Gul $M$, Uyarel $H$, Ergelen $M$, Karacimen D, Ugur $M$, Turer A. et al. The relationship between red blood cell distribution width and the clinical outcomes in non-ST elevation myocardial infarction and unstable angina pectoris: a 3-year follow-up. Coron Artery Dis 2012; 23(5):330-6.

8. Vayá A, Hernández V, Rivera L, Hernández JL, Lago A, España F, Bautista D. Red blood cell distribution width in patients with cryptogenic stroke. Clin Appl Thromb Hemost 2015; 21(3):241-5.

9. Patel KV, Ferrucci L, Ershler WB, Longo DL, Guralnik $J M$. Red blood cell distribution width and the risk of death in middle-aged and older adults. Arch Intern Med 2009; 169(5):515-523.

10. Sahin DY, Gür M, Elbasan Z, Özdo?ru I, Uysal OK, Kivrak A. et al. Mean platelet volume and extent and complexity of coronary artery disease in diabetic and nondiabetic patients with ST elevation myocardial infarction. Angiology 2013; 64(7):505-11.

11. Tanindi A, Topal FE, Topal F, Celik B. Red cell distribution width in patients with prehypertension and hypertension. Blood Press. 2012; 21(3):177-81.

12. Ozcan F, Turak $O$, Durak A, I?leyen A Uçar F, Gini? Z. et al. Red cell distribution width and inflammation in patients with non-dipper hypertension. Blood Press 2013; 22(2):80-5

13. Varol E, Akcay S, Icli A, Yucel H, Ozkan E, Erdogan $D$, Ozaydin M. Mean platelet volume in patients with prehypertension and hypertension. Clin Hemorheol Microcirc 2010; 45(1):67-72.

14. Inanc $T$, Kaya MG, Yarlioglues $M$, Ardic I, Ozdogru I, Dogan A. et al. The mean platelet volume in patients with non-dipper hypertension compared to dippers and normotensives. Blood Press 2010; 19(2):81-5.

15. Kurt RK, Aras Z, Silfeler DB, Kunt C, Islimye M, Kosar $O$. Relationship of red cell distribution width with the presence and severity of preeclampsia. Clin Appl Thromb Hemost. 2015;21(2):128-31

16. Abdullahi $H$, Osman A, Rayis DA, Gasim Gl, Imam AM, Adam I. Red blood cell distribution width is not correlated with preeclampsia among pregnant Sudanese women. Diagnostic Pathology 2014; 9:29.

17. Ceyhan T, Beyan C, Ba?er I, Kaptan K, Güngör $\mathrm{S}$, Ifran $\mathrm{A}$. The effect of pre-eclampsia on complete blood count, platelet count and mean platelet volume. Ann Hematol 2006; 85(5):320-2.

18. Freitas LG, Alpoim PN, Komatsuzaki F, Carvalho Md, Dusse LM. Preeclampsia: are platelet count and indices useful for its prognostic? Hematology 2013; 18(6):360-4.

19. ACOG practice bulletin. Diagnosis and management of preeclampsia and eclampsia. Number 33, January 2002. American College of Obstetricians and Ginecologists. Int J Gynaecol Obstet. 2002; 77(1):67-75.

20. H.J. Motulsky, Prism 5 Statistics Guide, 2007, GraphPad Software Inc., San Diego CA, www.graphpad. com

21. Tefferi A, Hanson CA, Inwards DJ. How to interpret and pursue an abnormal complete blood cell count in adults. Mayo Clin Proc 2005; 80:923-936.

22. Buttarello $M$. Laboratory diagnosis of anemia: are the old and new red cell parameters useful in classification and treatment, how? Int J Lab Hematol. 2016;38 Suppl1;123-132.

23. Huang $Y L$, Hu ZD, Liu SJ, Sun $Y$, Qin $Q$, Qin $B D$, et al. Prognostic value of red cell distribution width for patients with heart failure: a systematic review and meta-analysis of cohort studies. Plos One 2014; 9(8):e104861.

24. Vayá A, Sarnago A, Fuster O, Alis R, Romagnoli $M$. Influence of inflammatory and lipidic parameters on red blood cell distribution width in a healthy population. Clin Hemorheol Microcirc. 2015; 59(4):379-85.

25. Noris P, Klersy C, Gresele P, Giona F, Giordano P, Minoz P, Italian Gruppo di Studio delle Piastrine. Platelet size for distinguishing between inherited thrombocytopenia and immune thrombocytopenia: a multicentric, real life study. Br J Hematol. 2013;162(1);112-119.

26. Denghani $M R$, Taghipour-Sani $L$, Rezaei $Y$, Rostami R. Diagnostic importance of admission platelet volume indices in patients with acute chest pain suggesting acute coronary syndrome. Indian Heart J 2014; 66(6):622-8.

27. Martin JF, Trowbridge EA, Salmon G, Plumb J. The biological significance of platelet volume: its relationship to bleeding time, platelet thromboxane B2 production and megakaryocyte nuclear DNA concentration. Thromb Res 1983; 32:443-460.

28. Giles $H$, Smith RE, Martin JF. Platelet glycoprotein Ilb-IIla and size are increased in acute myocardial infarction. Eur J Clin Invest 1994; 24:69-72.

29. Pijnenborg R, Vercruysse $L$, Hanssens M. Fetalmaternal conflict, trophoblast invasion, preeclampsia, and the red queen. Hypertens Pregnancy 2008; 27:183-196.

30. Hung $\mathrm{TH}$, Burton GJ. Hypoxia and reoxygenation: a possible mechanism for placental oxidative stress in preeclampsia. Taiwan J Obstet Gynecol 2006; 45:189-200.

31. LaMarca BD, Gilbert J, Granger JP. Recent progress toward the understanding of the pathopysiology 
of hypertension during preeclampsia. Hypertension 2008; 51:982-988.

32. Kronborg CS, Gjedsted J, Vittinghus E, Hansen TK, Allen J, Knudsen UB. Longitudinal measurement of cytokines in pree-clamptic and normotensive pregnancies. Acta Obstet Gynecol Scand 2011; 90:791796.

33. Serin YS, Özcelik B, Bapbud M, Hüseyin K, Okur $D$, Erez R. Predictive value of tumor necrosis factor alpha (TNF-?) in preeclampsia. Euro Journ Obstet Gynecol 2002; 100:143-145.

34. Xiao JP, Yin YX, Gao YF, Lau S, Shen F, Zhao M, Chen $Q$. The increased maternal serum levels of IL- 6 are associated with the severity and onset of preeclampsia. Cytokine 2012; 60:856-860.

35. Yilmaz AZ, Yilmaz E, Kucikozan T. red blood cell distribution width: A simple parameter in preeclamspia.
Pregnancy Hypertens. 2016;6(4):285-287.

36. Sen-Yu, Chao X. Assessement of the relationship between red blood cell distribution width and pregnancy hypertension disease. J Obstet Gynaecol Res. 2016;42(10):1258-1262.

37. Buoro S, Carobene A, Seghezzi M, Manenti B, Dominoni $P$, Pacioni $A$, Ceriotti $F$, Ottomano C, Lippi $G$. Short-and medium- term biological variation estimates of red blood cell on reticulocyte parameters in healthy subjects. Clin Chem Lab Med. 2018.Jan $5 ; \mathrm{pii} / \mathrm{j} / \mathrm{cclm}$.

38. Hiderlink JM, Klinkenberg LJL, Aakre KM, de Wit $\mathrm{NCJ}$, Henskens YMC, van der Linden N, Bekers O Rennenberg RJMW, Koopmans RP, Meex SJR. Within-day biological variation and hour-to hour reference change values for hematological parameters. Clin Chem Lab Med 2017;55(7):1013-1024. 